\title{
1 Generation of Arctic-like Rabies Viruses Containing Chimeric Glycoproteins Enables Serological Potency
}

\section{Studies}

3

4 Emma M. Bentley ${ }^{1}$, Ruqiyo Ali ${ }^{1+}$, Daniel L. Horton ${ }^{2}$, Davide Corti ${ }^{3,4}$, Ashley C. Banyard ${ }^{5}$, Anthony R. Fooks ${ }^{5,6}$

5 and Edward Wright ${ }^{1 *}$

6

$7 \quad{ }^{1}$ Viral Pseudotype Unit, Faculty of Science and Technology, University of Westminster, 115 New Cavendish

8 Street, London, W1W 6UW, United Kingdom.

$9{ }^{2}$ School of Veterinary Medicine, University of Surrey, Daphne Jackson Road, Guildford, Surrey, GU2 7AL, United Kingdom.

${ }^{3}$ Humabs BioMed SA, 6500 Bellinzona, Switzerland.

${ }^{4}$ Institute for Research in Biomedicine, Università della Svizzera Italiana, 6500 Bellinzona, Switzerland.

${ }^{5}$ Wildlife Zoonoses and Vector Borne Diseases Research Group, Animal and Plant Health Agency, New Haw,

Addlestone, Surrey, KT15 3NB, United Kingdom.

${ }^{6}$ Institute for Infection and Immunity, St. George's Hospital Medical School, University of London, London,

+ Current address: MSAT Process development Medlmmune, Biopharmaceutical Development AstraZeneca,

Renaissance Way, Boulevard Industry Park, Liverpool L24 9JW, United Kingdom

* Corresponding author: Faculty of Science and Technology, University of Westminster, 115 New Cavendish

Street, London, W1W 6UW, United Kingdom. Tel.: +44 (0) 203506 414; E-mail address:

wrighte@westminster.ac.uk 


\section{ABSTRACT}

Rabies viruses have the highest case fatality rate of any known virus and are responsible for an estimated 60,000 deaths each year. This is despite the fact that there are highly efficacious vaccines and postexposure prophylaxis available. However, while it is assumed these biologics provide protection against all rabies virus isolates, there are certain subdivisions of RABV lineages, such as within the Arctic-like RABV (AL rabies virus lineage, where data is limited and thus the potency of existing biologics has not been thoroughly assessed. By fusing the Arctic-like rabies virus envelope glycoprotein ecto- and transmembrane domains with the vesicular stomatitis virus cytoplasmic domain, a high titre $\left(7.7 \times 10^{5}-6.1 \times 10^{6} \mathrm{RLU} / \mathrm{ml}\right)$ pseudotyped virus was generated that was subsequently used in a pseudotyped virus neutralisation assay. These results showed that Arctic-like rabies viruses are neutralised to human, canine and feline vaccines and human post-exposure prophylaxis and this was not influenced by the swapping of the cytoplasmic domains (CVS-11 vs CVS-11etmVSVc; $r=0.99, p<0.0001$ ). This study supports the concept that rabies virus vaccines and newly identified mAbs are able to neutralise rabies virus variants that cluster in a monophyletic clade, referred to as phylogroup I lyssaviruses. 
43

\section{INTRODUCTION}

Rabies, a neglected zoonotic disease caused by members of the Lyssavirus genus, poses a significant public health threat with a near $100 \%$ case fatality rate in infected individuals who do not receive pre or postexposure prophylaxes (Fooks et al., 2014). Globally, rabies virus (RABV) is accountable for an estimated 60,000 human deaths per year and the highest mortality rate of any other zoonotic disease when licenced vaccines and post-exposure prophylaxes are not administered (Fooks et al., 2014). Serological studies, monitoring responses to pre- and post-exposure prophylaxis and undertaking widespread sero-surveillance, are vital aspects for the implementation of control programmes to lower rabies incidence (Banyard et al., 2013; Brookes et al., 2005; Wright et al., 2009). However as many rabies-endemic areas are in the developing world, countries lack the infrastructure to be able to undertake these routine serological techniques (Banyard et al., 2013).

Fourteen members of the Lyssavirus species are classified, with RABV being the prototype species (Dietzgen et al., 2011). Two putative members of the Lyssavirus genus remain to be characterised and officially classified, Lleida bat lyssavirus (Ceballos et al., 2013) and Gannoruwa bat lyssavirus (Gunawardena et al., 2016). As infection with each species of the Lyssavirus genus causes a clinically indistinguishable disease the true burden of death from Lyssavirus species other than classical RABV is undefined. Arctic-like rabies viruses (AL RABV) form one of up to eight potential geographically and genetically distinct viral lineages of the RABV species (Kuzmin et al., 2008; Mansfield et al., 2006; Nadin-Davis et al., 2007; Troupin et al., 2016). Endemic across the Middle East and Asia, AL RABV is likely responsible for a significant proportion of rabies cases in these regions, which is proposed to result in greater than 20,000 human fatalities each year in India alone (Sudarshan et al., 2007). Yet due to inadequate reporting systems and a weak healthcare infrastructure across this region, it is likely the true burden of rabies is far higher (Banyard et al., 2013; Pant et al., 2013). This lack of accurate data has led to the low prioritisation of control programmes by policy makers and public health professionals (Fooks et al., 2014; Sudarshan et al., 2007). Domestic dogs are the predominant transmission vector in human cases, and as the annual economic cost of canine rabies alone is estimated to be 8.6 billion USD, the economic and societal implications of endemic rabies are severe 
(Hampson et al., 2015; Pant et al., 2013). Along with the implementation of control programmes to limit rabies incidence, it is also important to undertake a comprehensive analysis of currently circulating RABVs and monitor for the emergence of new variants (Matsumoto et al., 2013). While there is no evidence to indicate that AL RABV has an altered pathogenicity the protection afforded by vaccines and antivirals has not been specifically addressed. Thus it is important to fully understand the public health threat posed by this AL RABV lineage.

Pseudotyped virus (PV), a replication defective viral particle acting as a surrogate for live virus, has been used in a range of applications including serological assays and as vaccine immunogens (Mather et al., 2013; Temperton et al., 2015). The development of a PV neutralisation assay (PVNA) for the measurement of anti-rabies virus neutralising antibodies (VnAbs) in vaccine recipients, along with further large scale infield sero-surveillance within a developing country has previously been described, providing sensitive and specific results which correlate with live virus assays and distinguishes between lyssavirus species (Temperton et al., 2015; Wright et al., 2008, 2009, 2010). As the use of PV allows neutralisation assays to be undertaken in biosafety level 1 or 2 laboratories, along with having a lower cost implication, the serological study of rabies can be expanded to resource-limited laboratories in regions where the virus is endemic.

While Lyssavirus isolates have previously pseudotyped efficiently, AL RABV pseudotypes fail to generate an adequate titre to allow use in downstream neutralisation assay studies. The flexibility of using a chimeric glycoprotein to produce recombinant, live-virus RABV has previously been demonstrated (Foley et al., 2000). This was further applied in a study showing pseudotyping efficiency could be increased by altering the envelope glycoprotein, replacing the cytoplasmic domain with that of a glycoprotein that pseudotypes highly effectively(Carpentier et al., 2011). This study has adapted this approach to produce a chimeric AL RABV envelope glycoprotein, increasing PV titre, and undertaking PVNA to assess vaccine and antiviral efficacy against this AL RABV lineage. 
RESULTS

\section{Chimeric AL RABV envelope glycoprotein construction and PV titre}

Chimeric envelope G constructs were generated for four AL RABV isolates (RV61, RV193, RV250 and

RV277), selected based on clinical significance (RV61) and reported poor growth in reference laboratory live viral cultures (RV193 and RV277; APHA, UK) and to represent three genetically distinct clades of the Arcticrelated lineage (Fig. 1). The cytoplasmic domain sequence of each G was replaced with that of CVS-11 or VSV G. The ecto-transmembrane (etm) domain was not altered. A chimeric CVS-11 G sequence with a VSV G cytoplasmic domain (CVS-11etmVSVC) was produced for use as an internal control. Fig. 2 depicts the chimeric envelope $\mathrm{G}$ sequences generated.

Lentiviral PV comprising the wildtype and chimeric envelope $\mathrm{G}$ constructs were produced, packaging either an emerald green fluorescent protein (emGFP) or firefly luciferase reporter gene by transfecting HEK 293T/17 cells. These were titrated onto permissive BHK-21 cells to determine the viral titres of the chimeric constructs in comparison to that achieved using wildtype envelope G. Luciferase reporter PV with a chimeric CVS-11 cytoplasmic domain envelope G caused a decrease $(-15.7$ fold, $p=0.2 ;-10.6$ fold, $p=$ $0.0007 ;-1.4$ fold,$p=0.7)$ or insignificant increase ( 1.2 fold; $p=0.7)$ in viral titre in comparison to the wildtype envelope G (Fig. 3). However, PV with a chimeric VSV cytoplasmic domain envelope G resulted in a significant increase ( 11.3 to 83.3 fold; $p<0.0005$ ) in viral titre for three of the AL RABV isolates and a small (1.1 fold; $p=0.3$ ) increase in viral titre for the RV250 isolate (Fig. 3).

To corroborate this observation, PV with an emGFP reporter gene were generated and used in similar infection studies. Importantly, the increase in titre was also observed by fluorescent microscopy (Fig. 4a) and the fold increase determined by flow cytometry (Fig. 4b) was in line with that observed for the luciferase reporter PV constructs.

\section{Current vaccines and biologicals neutralise Arctic-like rabies virus}


The increased PV titre achieved for the AL RABV isolates using a chimeric VSV cytoplasmic domain envelope G enabled serology studies to be undertaken via a PVNA, to assess the level of sero-conversion afforded by current vaccines and post-exposure prophylaxis. The receptor-binding domain and antigenic sites of the RABV envelope $G$ have been mapped to the ectodomain (Evans et al., 2012; Kuzmina et al., 2013); consequently switching the cytoplasmic domain to generate chimeric constructs should not influence the serological profile. Sequence comparison of etm domains of the AL RABV isolates and CVS-11 G shows high homology (Fig. 5). This analysis suggests the neutralisation profiles should be similar, yet as it is based on sequences alone it only serves as a crude estimate due to the disproportionate effects of individual amino acids on antigenic properties.

Initially, the chimeric envelope G PV were tested alongside wildtype CVS-11 G PV using the OIE standard reference dog serum at a concentration of $0.5 \mathrm{IU} / \mathrm{ml}$ and $\mathrm{WHO} 2^{\text {nd }}$ international human anti-rabies $\mathrm{Ig}$ reference serum ( $2 \mathrm{IU} / \mathrm{ml}$; prepared by NIBSC, UK) over a total of twelve doubling dilutions. Chimeric CVS11etmVSVC G PV recorded an $I C_{100}$ titre matching $\left(I C_{100}=80\right)$ or within one doubling dilution $\left(I C_{100}=269\right)$ of that for wildtype CVS-11 G PV for the OIE and WHO standards respectively (Fig. 6). Further to this, all AL RABV chimeric envelope G PV were neutralised at an equivalent or more potent level by each standard than that recorded for CVS-11 G PV.

Analysis of the neutralisation afforded against these AL RABV isolates by pre-exposure vaccination was undertaken by assessing a blinded panel of serum samples taken from RABV-vaccinated humans and domestic animals (dogs and cats) vaccinated as part of the UK PETS. The samples had previously been given a titre (IU/ml) using the fluorescent antibody virus neutralisation (FAVN) test method for detecting rabies specific antibodies, a score of $0.5 \mathrm{IU} / \mathrm{ml}$ by FAVN is considered the cut-off for adequate sero-conversion for protection (Cliquet et al., 1998; WHO, 2013). When un-blinded, four human serum samples (H1, H5, H6, H7) with VnAb levels of $0.03-0.1 \mathrm{IU} / \mathrm{ml}$, did not neutralise any PV tested (data not shown) and one sample with a VnAb level just below $0.5 \mathrm{IU} / \mathrm{ml}(\mathrm{H} 61,0.38 \mathrm{IU} / \mathrm{ml}$ ) neutralised each of the PVs (Fig. 7a). All samples with a VnAb titre above $0.5 \mathrm{IU} / \mathrm{ml}$ produced high levels of neutralisation for the CVS-11 and CVS- 

adequate animal serum samples produced a strong neutralising response (Fig. 7b). Of the four samples tested which had previously demonstrated VnAbs titres between $0.07-0.38 \mathrm{IU} / \mathrm{ml}$ on FAVN testing (Fig. 7b; PET-5531,-5545,-5734,-5896) a low level of PV neutralisation was detected (IC 100 titres of 12-57).

Biologics used for post-exposure prophylaxis (PEP) were shown to effectively neutralise all AL RABV PV.

Human rabies immunoglobulin (HRIG) samples were tested with a starting concentration of $2 \mathrm{IU} / \mathrm{ml}$, each

sample provided a potent level of neutralisation (Fig. 7C). Monoclonal antibody preparations, directed

against various neutralising antigenic sites on the RABV envelope $G$ and being considered for development 
172

173

174

175

176

177

178

180

181

182

183

184

185

186

187

188

189

190

191

192

193

194

195

196

197

198

\section{DISCUSSION}

Serological studies are required to define $\mathrm{VnAb}$ titres as part of vaccination and antiviral development and treatment schedules, while also allowing surveillance of the epidemiological spread of emerging viruses. As PV incorporate envelope proteins identical to the wildtype virus in their envelope they are antigenically similar, mimic the action of live virus in neutralisation tests and have proven to be a safe, robust and flexible alternative for use in serological assays (Mather et al., 2013; Temperton et al., 2015). The PVNA can be undertaken in containment level 1 and 2 laboratories as it does not require the handling of live virus, as opposed to other rabies virus neutralisation tests. The range of reporter genes and removal of the need for cold-chain storage make the PVNA an accessible and lower cost alternative to conventional techniques.

Further to this, using a CVS-11 G PV, the PVNA proved to be $100 \%$ specific and equally sensitive to the WHO and OIE endorsed FAVN method of rabies VnAb detection (Wright et al., 2008, 2009). This study further substantiates its use by demonstrating the inherent flexibility of the platform, allowing manipulation of the envelope $\mathrm{G}$ to increase PV titre, permitting serological studies to determine the protection conferred by vaccines and antivirals against AL RABV isolates. While the PVNA is primarily a research tool at this time, it has previously been used in clinical trials to assess vaccines (Ewer et al., 2016; Ledgerwood et al., 2010).

Chimeric AL RABV envelope G sequences were constructed with either a CVS-11 or VSV G cytoplasmic domain in an attempt to increase PV titre. Both CVS-11 and VSV G routinely produce high titre PV. Only the chimeric VSV cytoplasmic domain envelope G resulted in a significant increase in PV titre for three of the AL RABV isolates. The lower increase in titre for the RV250 isolate is thought to be attributed to a difference in its glycoprotein structure, phylogenetic analysis showed greater sequence homology between the other isolates which formed a separate cluster. Previously, the use of a chimeric CVS (B2C strain) envelope G with a VSV cytoplasmic domain was described (Carpentier et al., 2011), reporting a two fold increase in titre; matching that observed for the CVS-11etmVSVC G used within this study. The mechanism behind this effect remains to be fully elucidated, yet several studies have described that the assembly of viable virions requires a direct or indirect interaction between the lentiviral matrix protein and envelope protein cytoplasmic domain (Cosson, 1996; Freed, 1998; Sandrin et al., 2004; Yu et al., 1992). Thus it is possible the 
cytoplasmic domain of VSV G interacts more effectively with the lentiviral matrix protein compared to that of CVS-11 G. Alternatively, it has been suggested a truncated or shorter cytoplasmic domain, as with VSV G, may cause a reduced steric hindrance or allow incorporation into lentiviral particles independent of matrix protein interaction (Freed \& Martin, 1995). This is further supported by the report that truncation of the measles virus fusion (F) protein cytoplasmic domain lead to an increased PV titre (Frecha et al., 2008).

Importantly, the regions of the VSV G defined within the literature differ due to the predictive nature of structural models. In this study, the VSV G cytoplasmic domain followed that used by Carpentier et al. (2011), as defined by Roche et al. (2006). However, caution is needed when designing chimeric sequences as alterations to some regions may result in loss of function, in particular the transmembrane domain which is involved in viral fusion and has more variability in the reported sequence (Cleverley $\&$ Lenard, 1998).

While current vaccines provide protection against RABV, the high level of sequence identity between the $A L$ RABV isolates and CVS-11 G is not sufficient to definitively predict their neutralisation profile, as the effect of individual amino acid substitutions on antigenic variation has in some cases proven substantial (Horton et al., 2010). Also, with the advent of $m A$ bs for PEP, point mutations within the binding sites of $m A$ Abs can result in viral escape from neutralisation and thus the identification of these critical residues, assessing the neutralisation of generated escape viruses, forms a vital aspect in the development of effective, broadly neutralising, therapeutics (Bakker et al., 2005; Marissen et al., 2005). Direct measures of antigenic variation by serology are fundamental yet can prove difficult to quantify. The use of antigenic cartography has added power to the interpretation of antigenic data, enabling the generation of an antigenic map for a global panel of lyssaviruses, instrumental for predicting antigenicity based on the envelope $G$ gene sequence (Horton et al., 2010). The PVNA platform has previously been used in the collection of antigenic data in a cross-species comparison of lyssavirus neutralisation, showing suitability as a high-throughput screening method to complement quantification of antigenic differences (Wright et al., 2008, 2009). This study further supports use of the PVNA, demonstrating inherent flexibility in the creation of chimeric viral 
envelope protein $\mathrm{PV}$ without disruption to the neutralisation profile and therefore the envelope protein

function. This enabled confirmation of sero-conversion, and by extrapolation, protection afforded by current vaccines and prophylaxis against the AL RABV isolates.

The AL RABV isolates were found to be effectively neutralised by human and mammalian serum samples, conferring adequate protection by current pre-exposure vaccine formulations. As more than $99 \%$ of human rabies cases occur following contact with rabid dogs, the control of rabies within this population is of high priority (Banyard et al., 2013; WHO, 2013). Mass vaccination campaigns targeting dog populations are highly effective and thus monitoring levels of protection afforded by animal vaccine formulations is of equal importance to the prevention of human rabies infections. All licenced vaccine preparations are derived from inactivated preparations of classical RABV, which has shown to confer protection against viruses in phylogroup I but offer limited or no protection against those in phylogroups II and III (Evans et al., 2012; Fooks, 2004; Hanlon et al., 2005). Since AL RABV is a lineage of classical RABV, the protection observed follows this accepted consensus and therefore, even though the isolates of rabies causing cases in the regions where AL RABV circulate are not fully characterised, unexplained vaccine failures have not been reported. However, due to poor growth of these AL RABV isolates in live viral cultures, which could suggest a different structure of the $G$ protein, and the implication of one isolate in a transplant-associated rabies outbreak in Germany (Ross et al., 2015), it was important to be able to undertake serological evaluation. Further studies into cross-protection of rabies vaccines against more divergent lyssaviruses, such as those within phylogroups II and III, using this PVNA could assist in the development of a more broadly cross reactive vaccine formulation.

PEP regimes have long been effective in preventing rabies virus infection in the event of exposure. For previously un-vaccinated individuals this consists of wound cleansing, vaccination and the administration of rabies immunoglobulin (RIG) to provide passive immunity in the interval before vaccine induced active immunity is achieved (Fooks et al., 2014). RIG of human (H) or equine (E) origin is available. While HRIG is preferred due to its longer half-life, it is expensive compared to the more immunogenic ERIG, which limits 
253

254

its use in the developing world; yet both are in short supply (WHO, 2013). The AL RABV isolates were neutralised by all HRIG preparations, however alternative means of PEP are now being sought by the development of mAb cocktails. Here we tested four mAbs, RVC20 and CR57, and RVC58 and CR4098, which target antigenic site I and III respectively of the RABV G (Bakker et al., 2005; De Benedictis et al., 2016; Marissen et al., 2005). In order to meet WHO guidelines, which suggest RABV PEP should contain at least two antibodies to lower the probability of immune escape, CR57 and CR4098 have been combined into the CL184 mAb cocktail and undergone phase II clinical trials (Bakker et al., 2008; Nagarajan et al., 2014; WHO, 2013). In this study, each mAb effectively neutralised the AL RABV isolates, which can further serve as an indication that both antigenic sites are highly conserved across the AL RABV lineage.

Ultimately, the flexibility of using PV demonstrated within this study can be further extended. The generation of antigenic escape mutant envelope protein for incorporation into the PV platform will enable evaluation of mAb cocktails undergoing development. Likewise, switching of epitopes between lyssavirus envelope $\mathrm{G}$ can allow further cross neutralisation studies to be undertaken, an important aspect in vaccine design. The ability to switch domains of the lyssavirus envelope $G$ has already been explored, highlighting the potential for use in antigenic studies (Jallet et al., 1999). This will enable the level of protection afforded against other divergent lyssaviruses in phylogroup II and III to be evaluated, of great interest from a public health perspective due to their unknown disease burden.

Using the approach of generating a chimeric envelope glycoprotein with a VSV cytoplasmic domain resulted in high titre PV without affecting their neutralisation profile. These data also provide further evidence of the flexibility pseudotyped virus-based assays provide when undertaking serological studies of highly pathogenic viruses. In conclusion, it was determined the AL RABV isolates are neutralised by available vaccines and post-exposure prophylaxis. 


\section{METHODS}

279

280

281

282

283

284

285

\section{Cell lines}

Human embryonic kidney 293T clone-17 cells (HEK 293T/17; ATCC CRL-11268) were used for PV production and subsequent titration and PVNA were undertaken using baby hamster kidney-21 clone-13 cells (BHK-21; ATCC CCL-10). Both cell lines were cultured in Dulbecco's Modified Eagle Medium (DMEM) supplemented with $10 \%$ foetal calf serum (FCS) and $1 \%$ penicillin/streptomycin with $5 \% \mathrm{CO}_{2}$.

\section{Viruses and cloning of chimeric envelope glycoprotein}

The four AL RABV envelope glycoprotein $(G)$ genes used in this study were amplified from viral RNA of India.human.87.RV61 (KU534939), Pakistan.dog.89.RV193 (KU534940), Russia.squirrel.RV250 (KU534941) and Pakistan.goat.RV277 (KU534942) and cloned into the pl.18 expression vector (Cox et al., 2002). The challenge virus standard-11 (CVS-11) (Wright et al., 2008) and vesicular stomatitis virus (VSV; a gift from Didier Trono, Adgene plasmid \# 12259) G genes have previously been described and were used to produce control pseudotyped virus in this study.

Chimeric envelope $\mathrm{G}$ were generated by overlap extension polymerase chain reaction (PCR) (Heckman \& Pease, 2007). Specific primers, designed based on the envelope G gene sequences, were used to separately amplify DNA fragments encoding the ecto- and transmembrane domain (etm) and cytoplasmic domain (c) portions of the chimeric constructs using the proofreading enzyme AccuPrime Pfx SuperMix (Life Technologies, UK). Primers are listed in Table S1. A further PCR was used to bring the entire open reading frame together utilising the overlapping complementary regions initially introduced. Once amplified, unique restriction sites introduced by the primers at the $5^{\prime}$ and $3^{\prime}$ ends were used to clone the chimeric sequences into the pl.18 expression plasmid. Clones containing the correct insert were identified by restriction enzyme digest and confirmed by Sanger sequencing.

\section{Pseudotyped virus production and titration}


Lentiviral pseudotyped virus production followed the transfection protocol previously described (Wright et al., 2009). Briefly, the HIV gag-pol construct p8.91 and firefly luciferase reporter construct pCSFLW or emerald green fluorescent protein (emGFP) reporter construct pCSemGW (kindly provided by University College London (UCL), UK) (Cubitt et al., 1998) were transfected concurrently with plasmid expressing the appropriate envelope G into HEK 293T/17 cells using Fugene-6 (Promega, UK) or polyethylenimine (PEI) (Sigma, UK) transfection reagent. Supernatant was harvested 48 and $72 \mathrm{~h}$ post-transfection and filtered through a $0.45 \mu \mathrm{m}$ filter, storing long-term at $-80^{\circ} \mathrm{C}$.

Titration of pseudotyped virus aliquots carrying the firefly luciferase reporter gene was performed by transducing BHK-21 cells in a 96-well plate $\left(2 \times 10^{4}\right.$ cells/well $)$ with serially diluted pseudotype supernatant in quadruplicate. Following 48h incubation, cell luminescence was read using the Bright-Glo assay (Promega, UK) and GloMax-Multi+ microplate luminometer (Promega, UK) with titres expressed as relative luminescence units per $\mathrm{ml}(\mathrm{RLU} / \mathrm{ml})$ or $50 \%$ tissue culture infective dose per $\mathrm{ml}\left(\mathrm{TCID}_{50} / \mathrm{ml}\right)$. Likewise, titration of virus with emGFP reporter gene involved transducing BHK-21 cells in duplicate with doubling dilutions of pseudotype supernatant. emGFP positive cells were visualised using a fluorescent microscope and counted using a Dako CyAn ADP cytometer (Beckman Coulter, UK).

\section{Serum and mAb samples}

The World Organisation for Animal Health (OIE) standard dog reference serum at a concentration of 0.5 international units per $\mathrm{ml}(\mathrm{IU} / \mathrm{ml})$ and WHO $2^{\text {nd }}$ international human anti-rabies $\mathrm{Ig}$ reference serum (2 IU/ml; prepared by National Institute for Biological Standards and Control (NIBSC), UK) were used as positive controls. A range of sera $(n=20)$ from RABV-vaccinated humans (Rabipur, Novartis) and dogs and cats (Rabvac, Fort Dodge; Nobivac, Intervet; Rabisin, Merial; Quantum, Schering Plough) enrolled in the UK pet travel scheme (PETS) were used (Ramnial et al., 2010). Human monoclonal antibody (mAb) samples were produced as described in (De Benedictis et al., 2016) and commercial rabies immunoglobulin (RIG) released for the European market were kindly provided by NIBSC, UK. 
All samples were titrated in 2-fold serial dilutions. All experiments were undertaken at least in duplicate,

332 where the titre varied by more than one doubling dilution it was repeated and the geometric mean

recorded, as per standard serological practice (Bresson et al., 2006).

\section{Neutralisation assays}

336 The pseudotyped virus $\mathrm{TCID}_{50}$ value was calculated using the end point method (Condit, 2001). In a 96-well

337 plate $50 \times \mathrm{TCID}_{50}$ pseudotyped virus was incubated with sera in duplicate for $1 \mathrm{hr}$ at $37^{\circ} \mathrm{C}\left(5 \% \mathrm{CO}_{2}\right)$ before

338 the addition of $1 \times 10^{4}$ BHK-21 cells. After a further 48 hrs incubation cell media was removed and a 50:50 mix of Bright-Glo reagent (Promega, UK) and fresh media added. Luciferase activity was detected on a GloMax-Multi+ microplate luminometer (Promega, UK) and IC 100 end-point titres recorded.

343 Analysis of 96 RABV glycoprotein sequences (1575 nucleotides) was inferred using MEGA6, with a GTR

344 substitution model, gamma distribution of rate variation sites a proportion of invariant sites (GTR+G+I).

345 Established lineages were illustrated and all except the Arctic-related viruses collapsed for clarity. Bootstrap 


\section{ACKNOWLEDGEMENTS}

348 We thank Greg Towers (UCL) for providing the pCSemGW plasmid and Giada Mattiuzzo (NIBSC) for

349 supplying the RIG samples used within this study. This work was supported by the Department for

350 Environment Food and Rural Affairs (Defra); Scottish Government and Welsh Government (grant number

351 SE0431).

352

353

\section{CONFLICTS OF INTEREST}

354

Davide Corti is employed by Humabs Biomed, which is developing rabies monoclonal antibodies. Ruqiyo Ali

355

undertook this work while an MSc student at the University of Westminster but has since taken up

356

employment at AstraZeneca.

357 


\section{REFERENCES}

Bakker, A. B. H., Python, C., Kissling, C. J., Pandya, P., Marissen, W. E., Brink, M. F., Lagerwerf, F., Worst, S., van Corven, E. \& other authors. (2008). First administration to humans of a monoclonal antibody cocktail against rabies virus: Safety, tolerability, and neutralizing activity. Vaccine 26, 5922-5927.

Bakker, A. B. H., Marissen, W. E., Arjen, R., Rice, A. B., Weldon, W. C., Niezgoda, M., Hanlon, C. a, Thijsse, S., Backus, H. H. J. \& other authors. (2005). Novel Human Monoclonal Antibody Combination Effectively Neutralizing Natural Rabies Virus Variants and Individual In Vitro Escape Mutants Novel Human Monoclonal Antibody Combination Effectively Neutralizing Natural Rabies Virus Variants and Individual In. J Virol 79, 9062-9068.

Banyard, A. C., Horton, D. L., Freuling, C., Müller, T. \& Fooks, A. R. (2013). Control and prevention of canine rabies: the need for building laboratory-based surveillance capacity. Antiviral Res 98, 357-64. Elsevier B.V.

Banyard, A. C. \& Fooks, A. R. (2017). The impact of novel lyssavirus discovery. Microbiol Aust 38, 17-21.

De Benedictis, P., Minola, A., Nodari, E. R., Aiello, R., Zecchin, B., Salomoni, A., Foglierini, M., Agatic, G., Vanzetta, F. \& other authors. (2016). Development of broad-spectrum human monoclonal antibodies for rabies post-exposure prophylaxis. EMBO Mol Med 8, 407-421.

Bresson, J.-L., Perronne, C., Launay, O., Gerdil, C., Saville, M., Wood, J., Höschler, K. \& Zambon, M. C. (2006). Safety and immunogenicity of an inactivated split-virion influenza A/Vietnam/1194/2004 (H5N1) vaccine: phase I randomised trial. Lancet 367, 1657-64.

Brookes, S. M., Parsons, G., Johnson, N., McElhinney, L. M. \& Fooks, a R. (2005). Rabies human diploid cell vaccine elicits cross-neutralising and cross-protecting immune responses against European and Australian bat lyssaviruses. Vaccine 23, 4101-9.

Carpentier, D. C. J., Vevis, K., Trabalza, A., Georgiadis, C., Ellison, S. M., Asfahani, R. I. \& Mazarakis, N. D. (2011). Enhanced pseudotyping efficiency of HIV-1 lentiviral vectors by a rabies/vesicular stomatitis virus chimeric envelope glycoprotein. Gene Ther 19, 761-74. Nature Publishing Group.

Ceballos, N. A., Morón, S. V., Berciano, J. M., Nicolás, O., López, C. A., Juste, J., Nevado, C. R., Setién, Á. A. \& Echevarría, J. E. (2013). Novel lyssavirus in bat, Spain. Emerg Infect Dis 19, 793-795.

Cleverley, D. Z. \& Lenard, J. (1998). The transmembrane domain in viral fusion: essential role for a conserved glycine residue in vesicular stomatitis virus G protein. Proc Natl Acad Sci U S A 95, 34253430.

Cliquet, F., Aubert, M. \& Sagné, L. (1998). Development of a fluorescent antibody virus neutralisation test (FAVN test) for the quantitation of rabies-neutralising antibody. J Immunol Methods 212, 79-87.

Condit, R. C. (2001). Principles of Virology. In Fields Virol, 4th edn., pp. 19-51. Edited by D. M. Knipe \& P. M. Howley. Philadelphia: Lippincott Williams \& Wilkins.

Cosson, P. (1996). Direct interaction between the envelope and matrix proteins of HIV-1. EMBO J 1, 57835788.

Cox, R. J., Mykkeltvedt, E., Robertson, J. \& Haaheim, L. R. (2002). Non-lethal viral challenge of influenza haemagglutinin and nucleoprotein DNA vaccinated mice results in reduced viral replication. Scand $J$ Immunol 55, 14-23.

Cubitt, A. B., Woollenweber, L. a \& Heim, R. (1998). Understanding Structure-Function Relationships in the Aequorea victoria Green Fluorescent Protein. Methods Cell Biol 58, 19-30.

Dietzgen, R. G., Calisher, C. H., Kurath, G., Kuzmin, I. V., Rodriguez, L. L., Stone, D. M., Tesh, R. B., Tordo, N., Walker, P. J. \& other authors. (2011). Rhabdoviridae. In Virus Taxon Ninth Rep Int Comm Taxon Viruses. Edited by M. J. A. Andrew, M. Q. King, E. B. Carstens \& E. J. Lefkowitz. Oxford, UK: Elsevier. 
402

403

404

405

406

407

408

409

410

411

412

413

414

415

416

417

418

419

420

421

422

423

424

425

426

427

428

429

430

431

432

433

434

435

436

437

438

439

440

441

442

443

444

445

Evans, J. S., Horton, D. L., Easton, A. J., Fooks, A. R. \& Banyard, A. C. (2012). Rabies virus vaccines: is there a need for a pan-lyssavirus vaccine? Vaccine 30, 7447-54. Elsevier Ltd.

Ewer, K., Rampling, T., Venkatraman, N., Bowyer, G., Wright, D., Lambe, T., Imoukhuede, E. B., Payne, R., Fehling, S. K. \& other authors. (2016). A Monovalent Chimpanzee Adenovirus Ebola Vaccine Boosted with MVA. N Eng/ J Med 374, 1635-1646.

Foley, H. D., McGettigan, J. P., Siler, C. a, Dietzschold, B. \& Schnell, M. J. (2000). A recombinant rabies virus expressing vesicular stomatitis virus glycoprotein fails to protect against rabies virus infection. Proc Natl Acad Sci U S A 97, 14680-14685.

Fooks, A. (2004). The challenge of new and emerging lyssaviruses. Expert Rev Vaccines 3, 333-6.

Fooks, A. R., Banyard, A. C., Horton, D. L., Johnson, N., McElhinney, L. M. \& Jackson, A. C. (2014). Current status of rabies and prospects for elimination. Lancet 6736, 1-11. Elsevier Ltd.

Frecha, C., Costa, C., Nègre, D., Gauthier, E., Russell, S. J., Cosset, F.-L. \& Verhoeyen, E. (2008). Stable transduction of quiescent $T$ cells without induction of cycle progression by a novel lentiviral vector pseudotyped with measles virus glycoproteins. Blood 112, 4843-52.

Freed, E. O. (1998). HIV-1 gag proteins: diverse functions in the virus life cycle. Virology 251, 1-15.

Freed, E. O. \& Martin, M. a. (1995). Virion incorporation of envelope glycoproteins with long but not short cytoplasmic tails is blocked by specific, single amino acid substitutions in the human immunodeficiency virus type 1 matrix. J Virol 69, 1984-1989.

Gunawardena, P. S., Marston, D. A., Ellis, R. J., Wise, E. L., Karawita, A. C., Breed, A. C., Mcelhinney, L. M., Johnson, N., Banyard, A. C. \& Fooks, A. R. (2016). Lyssavirus in Indian Flying Foxes, Sri Lanka. Emerg Infect Dis 22, 8-11.

Hampson, K., Coudeville, L., Lembo, T., Sambo, M., Kieffer, A., Attlan, M., Barrat, J., Blanton, J. D., Briggs, D. J. \& other authors. (2015). Estimating the Global Burden of Endemic Canine Rabies. PLoS Negl Trop Dis 9, e0003709.

Hanlon, C. A., Kuzmin, I. V, Blanton, J. D., Weldon, W. C., Manangan, J. S. \& Rupprecht, C. E. (2005). Efficacy of rabies biologics against new lyssaviruses from Eurasia. Virus Res 111, 44-54.

Heckman, K. L. \& Pease, L. R. (2007). Gene splicing and mutagenesis by PCR-driven overlap extension. Nat Protoc 2, 924-32.

Horton, D. L., McElhinney, L. M., Marston, D. a, Wood, J. L. N., Russell, C. a, Lewis, N., Kuzmin, I. V, Fouchier, R. a M., Osterhaus, a D. M. E. \& other authors. (2010). Quantifying antigenic relationships among the lyssaviruses. J Virol 84, 11841-8.

Jallet, C., Jacob, Y., Bahloul, C., Drings, a, Desmezieres, E., Tordo, N. \& Perrin, P. (1999). Chimeric lyssavirus glycoproteins with increased immunological potential. $J$ Virol 73, 225-233.

Kuzmin, I. V, Hughes, G. J., Botvinkin, a D., Gribencha, S. G. \& Rupprecht, C. E. (2008). Arctic and Arcticlike rabies viruses: distribution, phylogeny and evolutionary history. Epidemiol Infect 136, 509-19.

Kuzmina, N., Kuzmin, I. V, Ellison, J. A. \& Rupprecht, C. E. (2013). Conservation of Binding Epitopes for Monoclonal Antibodies on the Rabies Virus Glycoprotein. J Antivir Antiretrovir 5, 37-43.

Ledgerwood, J. E., Costner, P., Desai, N., Holman, L., Enama, M. E., Yamshchikov, G., Mulangu, S., Hu, Z., Andrews, C. A. \& other authors. (2010). A replication defective recombinant Ad5 vaccine expressing Ebola virus GP is safe and immunogenic in healthy adults. Vaccine 29, 304-313.

Mansfield, K. L., Racloz, V., McElhinney, L. M., Marston, D. A., Johnson, N., R??nsholt, L., Christensen, L. S., Neuvonen, E., Botvinkin, A. D. \& other authors. (2006). Molecular epidemiological study of Arctic rabies virus isolates from Greenland and comparison with isolates from throughout the Arctic and Baltic regions. Virus Res 116, 1-10. 
Marissen, W. E., Kramer, R. A., Rice, A., Weldon, W. C., Niezgoda, M., Faber, M., Slootstra, J. W., Meloen, R. H., Clijsters-van der Horst, M. \& other authors. (2005). Novel rabies virus-neutralizing epitope recognized by human monoclonal antibody: fine mapping and escape mutant analysis. $J$ Virol 79, 4672-4678.

Mather, S., Scott, S., Temperton, N., Wright, E., King, B. \& Daly, J. (2013). Current progress with serological assays for exotic emerging/re-emerging viruses. Future Viro/ 8, 745-755.

Matsumoto, T., Ahmed, K., Karunanayake, D., Wimalaratne, O., Nanayakkara, S., Perera, D., Kobayashi, Y. \& Nishizono, A. (2013). Molecular epidemiology of human rabies viruses in Sri Lanka. Infect Genet Evol 18, 160-7. Elsevier B.V.

Nadin-Davis, S. a, Turner, G., Paul, J. P. V, Madhusudana, S. N. \& Wandeler, A. I. (2007). Emergence of Arctic-like rabies lineage in India. Emerg Infect Dis 13, 111-6.

Nagarajan, T., Marissen, W. E. \& Rupprecht, C. E. (2014). Monoclonal antibodies for the prevention of rabies?]: theory and clinical practice. Antib Technol J 4, 1-12.

Pant, G. R., Lavenir, R., Wong, F. Y. K., Certoma, A., Larrous, F., Bhatta, D. R., Bourhy, H., Stevens, V. \& Dacheux, L. (2013). Recent emergence and spread of an Arctic-related phylogenetic lineage of rabies virus in Nepal. PLoS Negl Trop Dis 7, e2560.

Ramnial, V., Kosmider, R., Aylan, O., Freuling, C., Müller, T. \& Fooks, a R. (2010). Quantitative risk assessment to compare the risk of rabies entering the UK from Turkey via quarantine, the Pet Travel Scheme and the EU Pet Movement Policy. Epidemiol Infect 138, 1114-1125.

Roche, S., Bressanelli, S., Rey, F. \& Gaudin, Y. (2006). Crystal Structure of the Low-pH Form of the Vesicular Stomatitis Virus Glycoprotein G. Science 313, 187-192.

Ross, R. S., Wolters, B., Hoffmann, B., Geue, L., Viazov, S., Grüner, N., Roggendorf, M. \& Müller, T. (2015). Instructive even after a decade: Complete results of initial virological diagnostics and re-evaluation of molecular data in the German rabies virus 'outbreak' caused by transplantations. Int J Med Microbiol 305, 636-643. Elsevier GmbH.

Sandrin, V., Muriaux, D., Darlix, J. \& Inserm, U. (2004). Intracellular Trafficking of Gag and Env Proteins and Their Interactions Modulate Pseudotyping of Retroviruses. J Virol 78, 7153-7164.

Sudarshan, M. K., Madhusudana, S. N., Mahendra, B. J., Rao, N. S. N., Ashwath Narayana, D. H., Abdul Rahman, S., Meslin, F.-X., Lobo, D., Ravikumar, K. \& Gangaboraiah. (2007). Assessing the burden of human rabies in India: results of a national multi-center epidemiological survey. Int J Infect Dis 11, 2935.

Temperton, N. J., Wright, E. \& Scott, S. D. (2015). Retroviral Pseudotypes - from scientific tools to clinical utility. Encycl Life Sci 1-21.

Troupin, C., Dacheux, L., Tanguy, M., Sabeta, C., Holmes, E. C., Bouchier, C., Vignuzzi, M., Duchene, S., Holmes, E. \& Bourhy, H. (2016). Large-Scale Phylogenomic Analysis Reveals the Complex Evolutionary History of Rabies Virus in Multiple Carnivore Hosts. PLoS Pathog 12, e1006041.

WHO. (2013). WHO Expert Consultation on Rabies - Second Report. Geneva.

Wright, E., Temperton, N. J., Marston, D. A., McElhinney, L. M., Fooks, A. R. \& Weiss, R. A. (2008). Investigating antibody neutralization of lyssaviruses using lentiviral pseudotypes: a cross-species comparison. J Gen Virol 89, 2204-13.

Wright, E., McNabb, S., Goddard, T., Horton, D. L., Lembo, T., Nel, L. H., Weiss, R. a, Cleaveland, S. \& Fooks, A. R. (2009). A robust lentiviral pseudotype neutralisation assay for in-field serosurveillance of rabies and lyssaviruses in Africa. Vaccine 27, 7178-86.

Wright, E., Hayman, D. T. S., Vaughan, A., Temperton, N. J., Wood, J. L. N., Cunningham, A. A., Suu-Ire, R., Weiss, R. A. \& Fooks, A. R. (2010). Virus neutralising activity of African fruit bat (Eidolon helvum) sera 
bioRxiv preprint doi: https://doi.org/10.1101/150300; this version posted June 15, 2017. The copyright holder for this preprint (which was not certified by peer review) is the author/funder, who has granted bioRxiv a license to display the preprint in perpetuity. It is made available under aCC-BY-NC-ND 4.0 International license.

against emerging lyssaviruses. Virology 408, 183-9. Elsevier Inc.

Yu, X., Yuan, X., Matsuda, Z., Lee, T. H. \& Essex, M. (1992). The matrix protein of human immunodeficiency virus type 1 is required for incorporation of viral envelope protein into mature virions. J Virol 66,

494 4966-4971.

495

496 


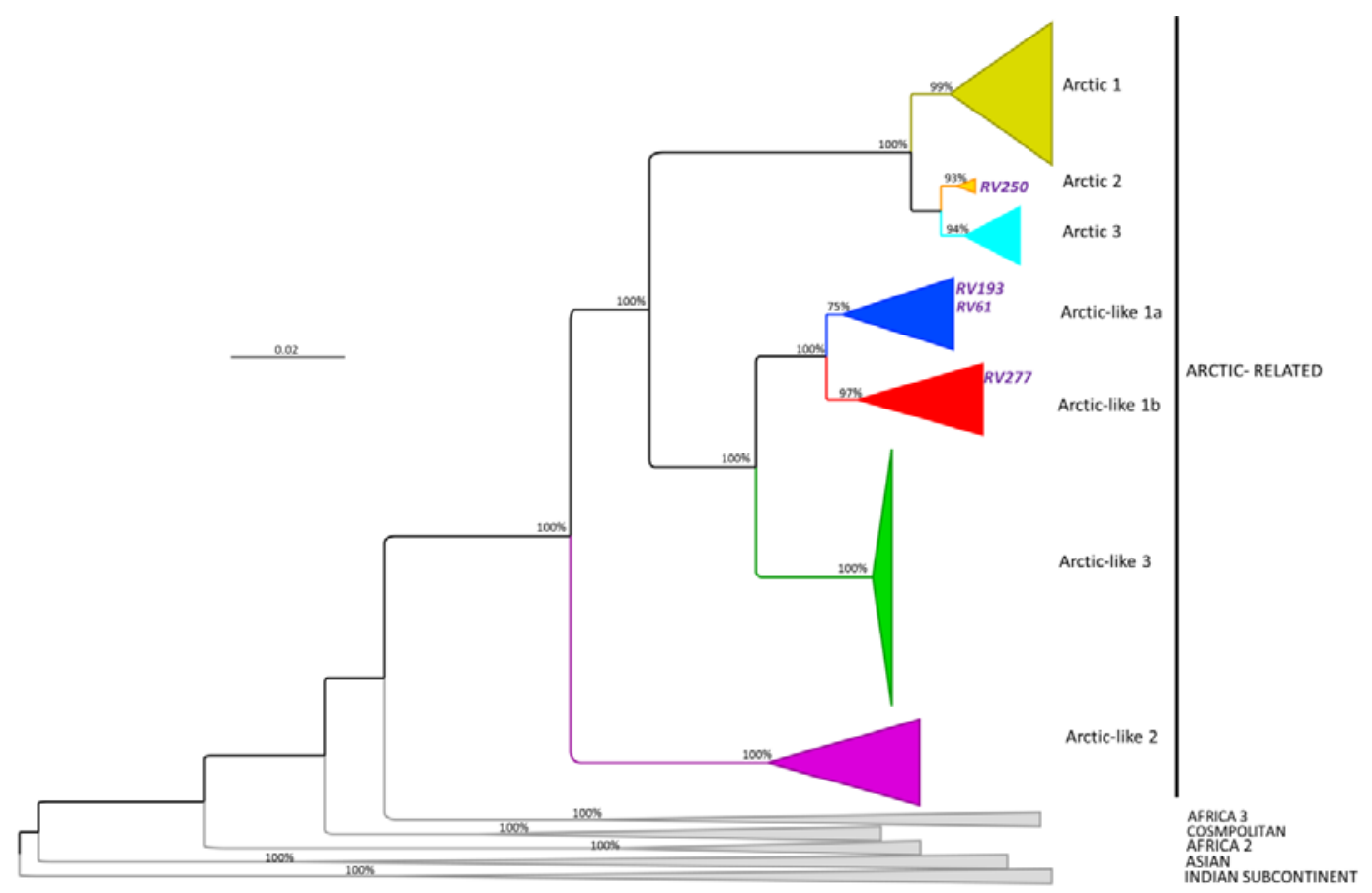

501 Positions of the viruses used in this study (RV61, RV193, RV250 and RV277) are indicated. 


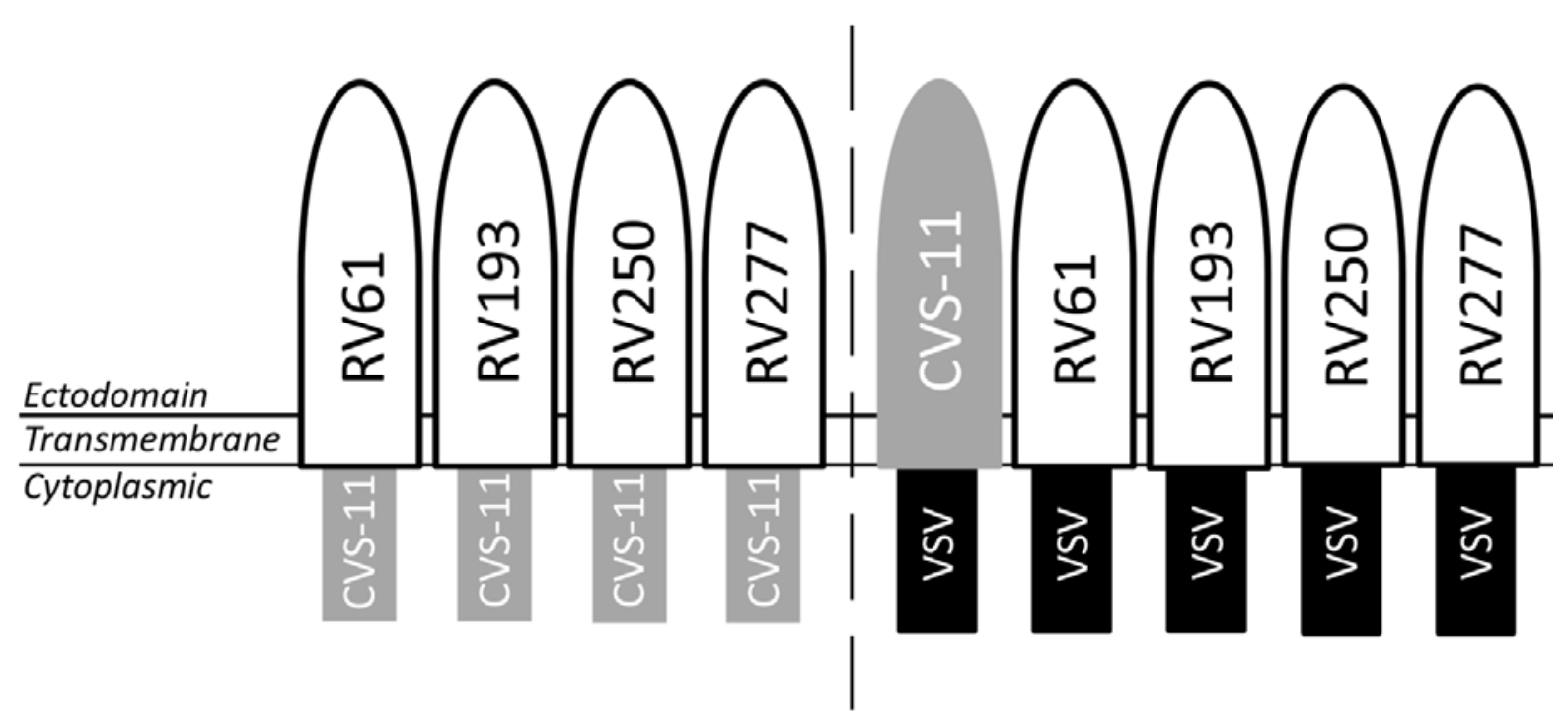

Fig. 2. Schematic of the chimeric envelope glycoprotein constructs generated. AL RABV and CVS-11 ecto- 


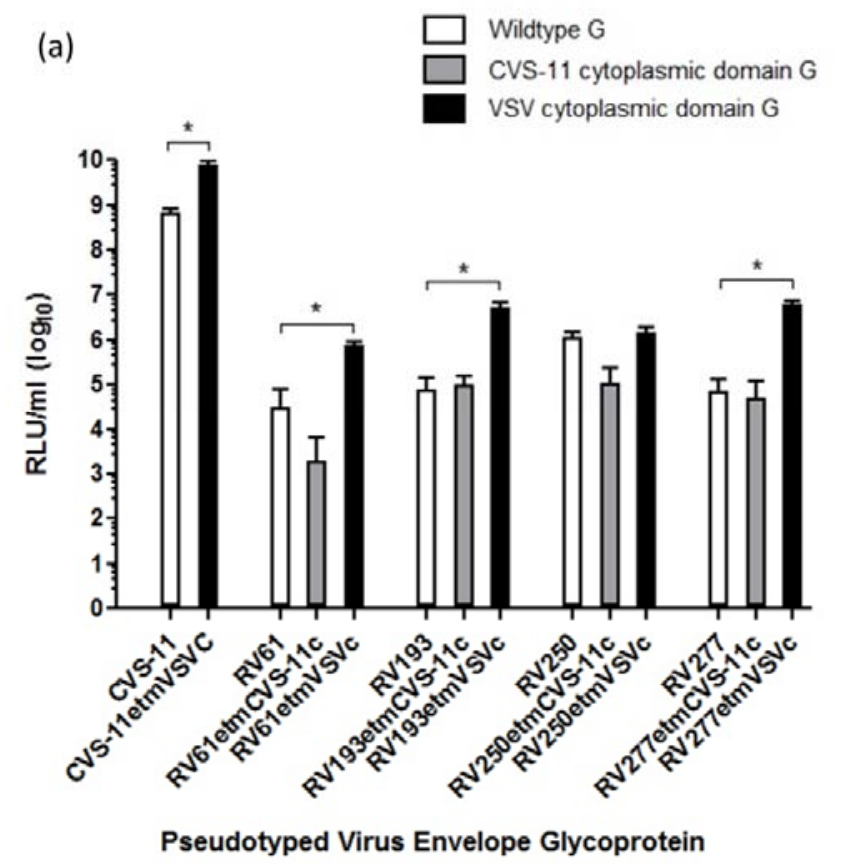

(b)

\begin{tabular}{cc}
\hline Chimeric G & Fold Increase \\
\hline CVS-11etmVSVc & 11.3 \\
RV61etmVSVc & 24.2 \\
RV193etmVSVc & 67.9 \\
RV250etmVSVc & 1.1 \\
RV277etmVSVc & 83.3 \\
\hline
\end{tabular}

Fig. 3. Comparison of viral titres using wildtype and chimeric envelope glycoproteins. (a) Aliquots of PV 
(a)
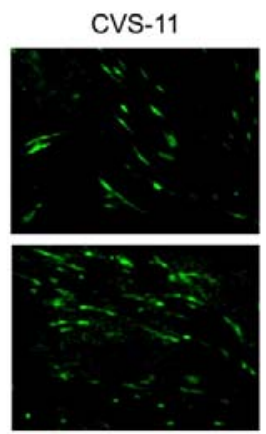

(b) CVS-11etmVSVc
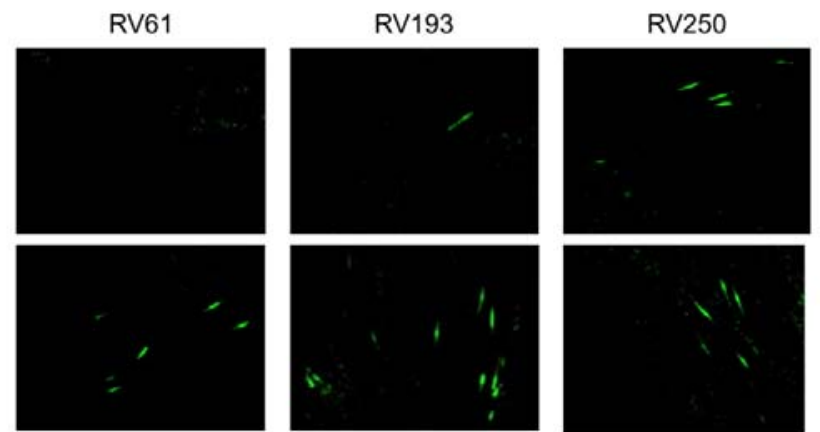

RV61etmVSVc

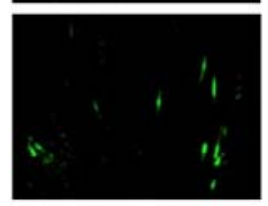

RV193etmVSVc

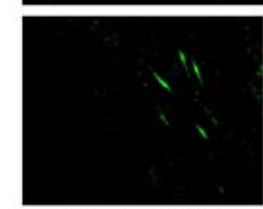

RV250etmVSVc
RV277

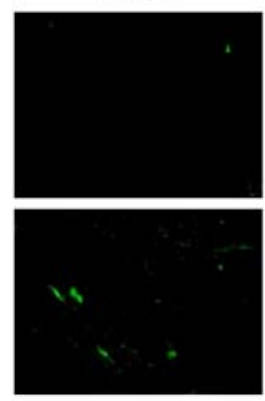

RV277etmVSVc

\begin{tabular}{c|ccccc}
$\begin{array}{c}\text { Fold } \\
\text { Increase }\end{array}$ & 2 & 55 & 27 & 8 & 19
\end{tabular}

515

516 Fig. 4. Viral titre comparison of PV bearing chimeric envelope glycoprotein and carrying an emGFP

517 reporter gene. (a) Fluorescent micrographs of BHK cells infected with wildtype or chimeric VSVc G PV. (b)

518 Fold increase in viral titre comparing chimeric VSVC G PV to wildtype stocks used in (a), determined by flow cytometry analysis. 
(a)

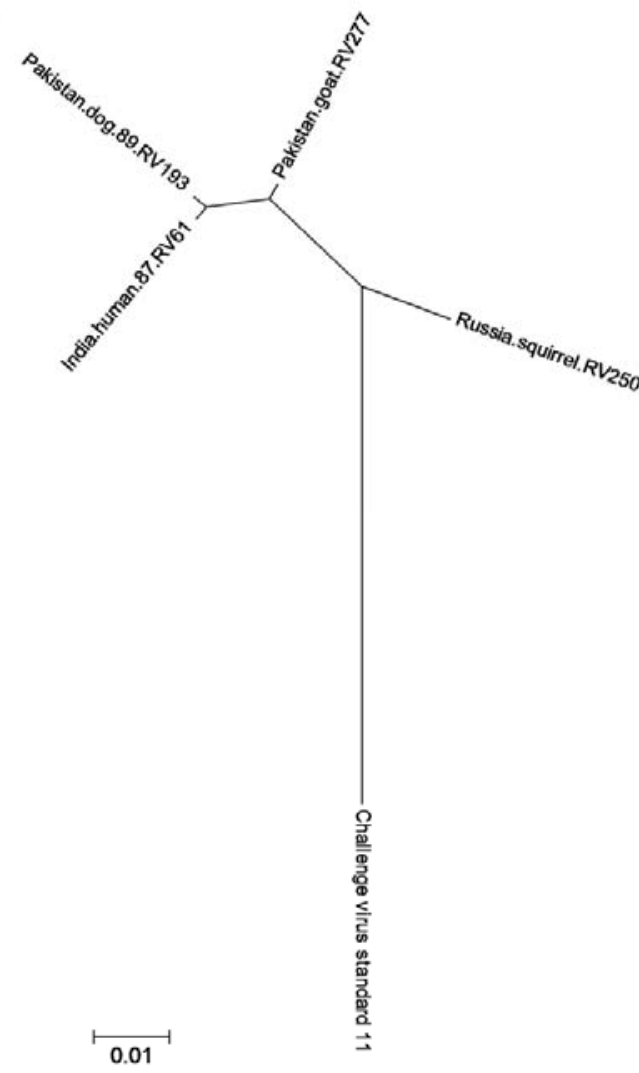

(b)

\begin{tabular}{|c|c|c|c|c|c|c|}
\hline & \multicolumn{6}{|c|}{ Amino acid (\% identity) } \\
\hline \multirow{6}{*}{ 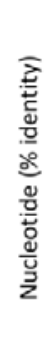 } & & CVS-11 & RV61 & RV193 & RV250 & RV277 \\
\hline & CVS- 11 & & 92 & 92 & 93 & 92 \\
\hline & RV61 & 87 & & 99 & 96 & 99 \\
\hline & RV193 & 87 & 99 & & 96 & 99 \\
\hline & $R V 250$ & 87 & 92 & 93 & & 97 \\
\hline & RV277 & 88 & 97 & 98 & 93 & \\
\hline
\end{tabular}

\section{Fig. 5. Degree of nucleotide and amino acid sequence identity between envelope glycoprotein etm}

523 domains of the rabies virus isolates within this study. (a) The radial phylogenetic tree scale corresponds to amino acid substitutions per site. (b) Nucleotide and amino acid percentage identities are shown. 
OIE Serum

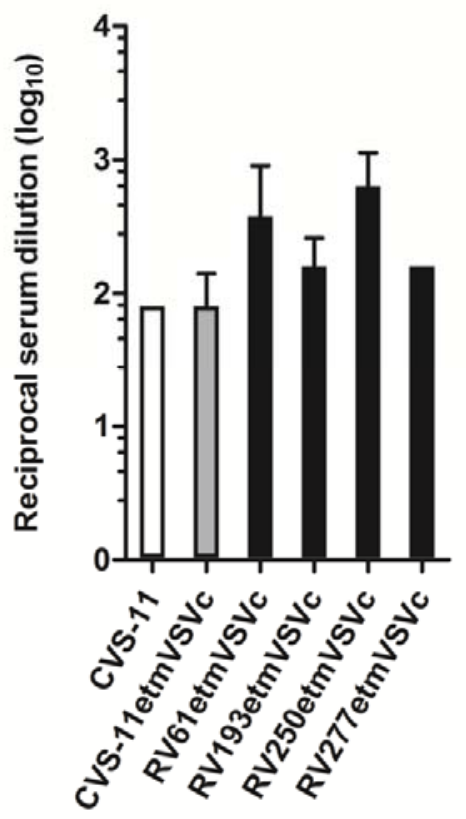

WHO Serum

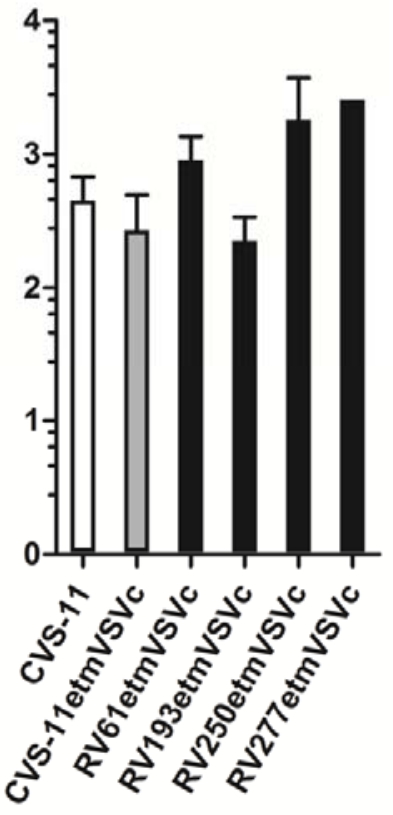

527 Fig. 6. Neutralisation of PV by OIE and WHO serum standards. The OIE is a standard reference dog serum

528 at $0.5 \mathrm{IU} / \mathrm{ml}$ and the $\mathrm{WHO}$ is the $2^{\text {nd }}$ international human anti-rabies $\mathrm{Ig}$ reference serum at $2 \mathrm{IU} / \mathrm{ml}$. Values are reported as $\mathrm{IC}_{100}$ endpoint reciprocal dilutions (geometric mean $\pm \mathrm{SD}$ ). Where error bars are absent, replicates produced the same $\mathrm{IC}_{100}$ endpoint dilution. 
(a)

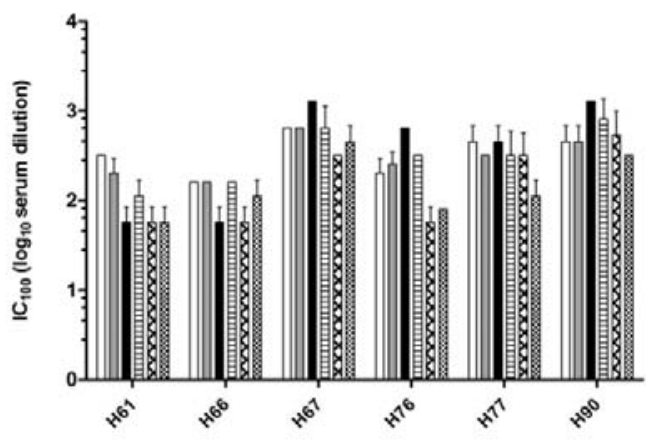

(c)

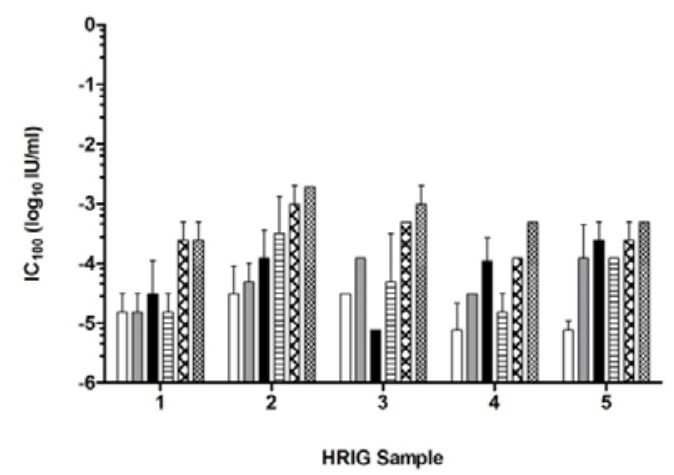

(b)

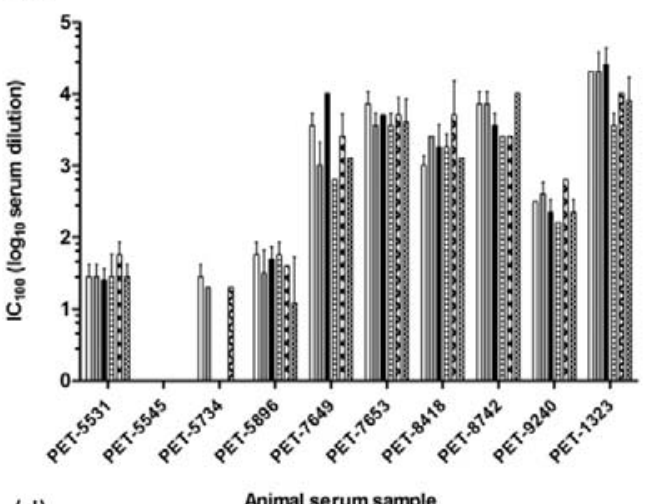

(d)

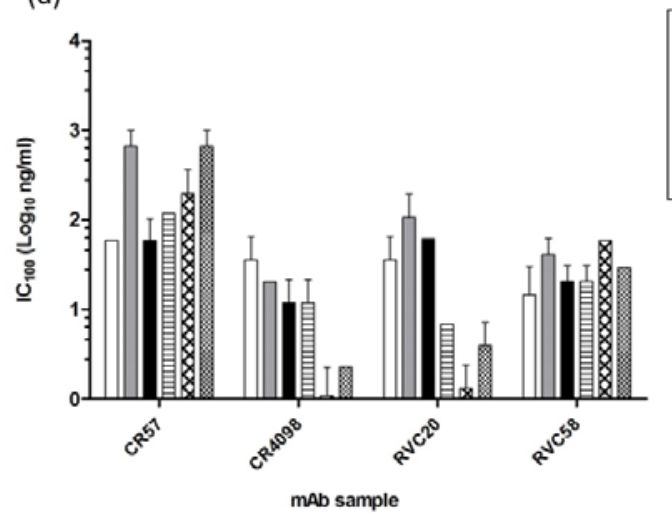

$\square$ CVS-11

CVS-11etmVSVc

- RVB1etmVSVc

$\boxminus$ RV193etmVSVc

XX RV250etmVSVe

RV277etmVSVc

\section{2}

Fig. 7. Neutralisation $I_{100}$ endpoint dilutions for human and animal serum samples, HRIG and mAb

samples are reported for CVS-11 and AL RABV G PV. (a) Human serum samples are from RABV vaccine

recipients, sample $\mathrm{H} 61$ has a titre of $0.38 \mathrm{IU} / \mathrm{ml}$ and the remaining samples a titre $>0.5 \mathrm{IU} / \mathrm{ml}$. IC 100 values

are reported as reciprocal serum dilutions. (b) Animal serum samples are from vaccinated dogs or cats, four

samples with titres between $0.07-0.38$ IU/ml (PET-5531,-5545,-5734,-5896) are shown. The remaining

samples have a titre $>0.5 \mathrm{IU} / \mathrm{ml}$ and $\mathrm{IC}_{100}$ values are reported as reciprocal serum dilutions. (c) $\mathrm{HRIG}$

samples were tested with a starting concentration of $2 \mathrm{IU} / \mathrm{ml}$. (d) $\mathrm{mAb}$ samples are derived against different

neutralising epitopes and were used at a starting concentration of $15 \mu \mathrm{g} / \mathrm{ml}$. All values are reported as the

dilution. 


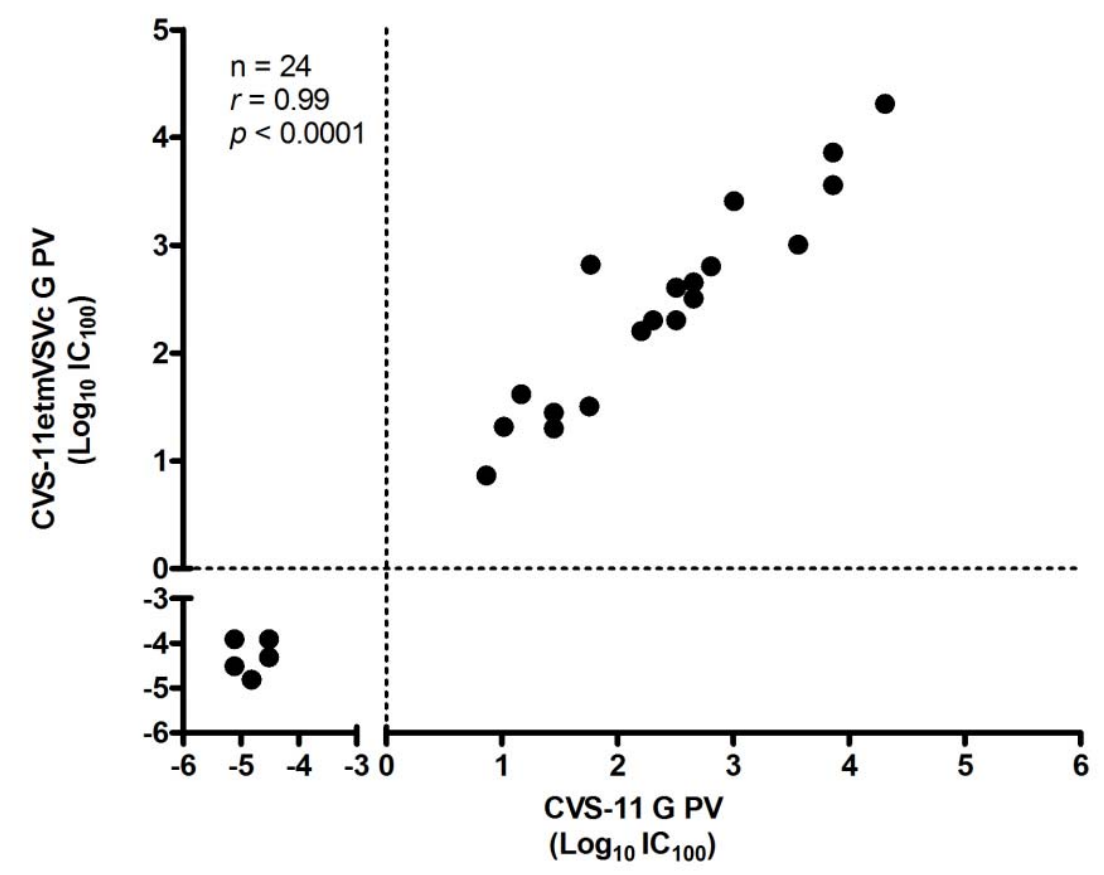

545 Fig. 8. Comparison of the neutralisation $\mathrm{IC}_{100}$ endpoint for wildtype CVS-11 G PV compared to chimeric

546 CVS-11etmVSVc G PV. A high correlation $(r)$ was observed. Pearson's product-moment correlation was

547 used to calculate $r$ and $p$ values.

548 
549 Table S1. Oligonucleotide primers used for PCR amplification and overlap extension PCR to create

550 chimeric envelope glycoprotein sequences.

\begin{tabular}{|c|c|c|}
\hline Glycoprotein Gene & Forward Primer $\left(5^{\prime} \rightarrow 3^{\prime}\right)$ & Reverse Primer $\left(5^{\prime} \rightarrow 3^{\prime}\right)$ \\
\hline CVS-11 & $\begin{array}{l}\text { GCGCGCGGTACCGCCACCATGGTTCCTCAAGT } \\
\text { TCTT }\end{array}$ & GCGCGCCTCGAGTTACAGTCTGATCTCACCTC \\
\hline RV61 & $\begin{array}{l}\text { GCGCGCGGTACCGCCACCATGGTTCCTCAAGT } \\
\text { TCTT }\end{array}$ & GCGCGCCTCGAGTCACAGTCTGGTCTCACC \\
\hline RV193 & $\begin{array}{l}\text { GCGCGCGGTACCGCCACCATGGTTCCTCAGGT } \\
\text { TCTT }\end{array}$ & GCGCGCCTCGAGTCACAGTCTGGTCTCACC \\
\hline RV250 & $\begin{array}{l}\text { GCGCGCGGTACCGCCACCATGGTTCCTCAAGC } \\
\text { TCTT }\end{array}$ & GCGCGCGAATTCTCACAGTCTGGTCTCACC \\
\hline RV277 & $\begin{array}{l}\text { GCGCGCGGTACCGCCACCATGGTTCCTCAGGT } \\
\text { TCTT }\end{array}$ & GCGCGCCTCGAGTCACAGTCTGGTCTCACC \\
\hline RV61etmCVS-11c & RV61 forward & CVS-11 reverse \\
\hline RV193etmCVS-11c & RV193 forward & CVS-11 reverse \\
\hline RV250etmCVS-11c & RV250 forward & GAGCGCGAATTCTTACAGTCTGATCTCACCTC \\
\hline RV277etmCVS-11c & RV277 forward & CVS-11 reverse \\
\hline CVS-11etmVSVc & CVS-11 forward & GATCATCTCGAGTTACTTTCCAAGTCGGTTCA \\
\hline RV61etmVSVc & RV61 forward & GATCATCTCGAGTTACTTTCCAAGTCGGTTCA \\
\hline RV193etmVSVc & RV193 forward & GATCATCTCGAGTTACTTTCCAAGTCGGTTCA \\
\hline RV250etmVSVc & RV250 forward & GATCATGAATTCTTACTTTCCAAGTCGGTTCA \\
\hline \multirow[t]{2}{*}{ RV277etmVSVc } & RV277 forward & GATCATCTCGAGTTACTTTCCAAGTCGGTTCA \\
\hline & Internal Forward Primer $\left(5^{\prime} \rightarrow 3^{\prime}\right)$ & Internal Reverse Primer $\left(5^{\prime} \rightarrow 3^{\prime}\right)$ \\
\hline RV61etmCVS-11c & ACATGTTGC AGAAGAGCCAAT & GGCTCTTCT GCAACATGTTAT \\
\hline RV193etmCVS-11c & ACATGTTGC AGAAGAGCCAAT & GGCTCTTCT GCAACATGTTAT \\
\hline RV250etmCVS-11c & GACATGTTGT AGAAGAGCCAA & GGCTCTTCT ACAACATGTCATT \\
\hline RV277etmCVS-11c & ACATGTTGC AGAAGAGCCAAT & GGCTCTTCT GCAACATGTCATT \\
\hline CVS-11etmVSVc & GACATGGTGC CGAGTTGGTATCCAT & TACCAACTCG GCACCATGTCATTAG \\
\hline RV61etmVSVc & AACATGTTGC CGAGTTGGTATCCAT & TACCAACTCG GCAACATGTTATTATG \\
\hline RV193etmVSVc & AACATGTTGC CGAGTTGGTATCCAT & TACCAACTCG GCAACATGTTATTATG \\
\hline RV250etmVSVc & GACATGTTGT CGAGTTGGTATCCAT & TACCAACTCG ACAACATGTCATTAAG \\
\hline RV277etmVSVc & GACATGTTGC CGAGTTGGTATCCAT & TACCAACTCG GCAACATGTCATTATG \\
\hline
\end{tabular}

551 